\title{
EL GÉNERO ARRABIDAEA (BIGNONIACEAE) EN ARGENTINA: NUEVOS REGISTROS, DISTRIBUCIÓN Y FENOLOGÍA
}

\author{
por MARIA MERCEDES ARBO'
}

Summary

The argentine Bignoniaceae include 23 genera and 56 species. Among the vines, Arrabidaea DC. is the genus with the highest number of native species. A.samydoïdes (Cham.) Sandwith, not known for Argentina, and A.triplinervia (DC.) Baill. ex Bureau, which was cited but not documented, are described and illustrated. A key is provided to identify the nine native species. A comparative analysis is made of their distribution in Argentina and their phenology.

Las Bignoniaceae argentinas incluyen 23 géneros y 56 especies, entre las que se cuentan varios árboles con maderas valiosas y numerosas lianas de flores muy bellas. Entre las lianas, el género con mayor número de representantes nativos es Arrabidaea DC. Este género incluye unas 70 especies de regiones tropicales y subtropicales. Generalmente está asociado con vegetación arbórea, en la selva alcanza los estratos más altos. La mayoría de las especies son polinizadas por abejas, y las semillas son diseminadas por el viento.

En la revisión de las Bignoniaceae argentinas, Fabris (1965) describió seis especies. Posteriormente, Sandwith (1968) sinonimizó Petastoma con Arrabidaea, agregándose de esta manera otra especie. Al revisar colecciones recientes realizadas en el Parque Nacional Iguazú por personal del Instituto de Botạnica del Nordeste, y material de herbarios argentinos, se encontró una especie de Arrabidaea que no estaba citada para la República Argentina: A.samydoïdes (Cham.) Sandwith. Tambiên se halló A.triplinervia (DC.) Baill. ex Bureau, que si bien fue citada para Misiones, sin indicación de localidad (Latzina, 1937; Devoto \& Rothkugel, 1942), no estaba documentada con ejem-

' Facultad de Ciencias Agrarias - Instituto de Botánica del Nordeste, C.C.209, 3400 Corrientes, Argentina. Email arbo@agr.unne.edu.ar plares de herbario; Fabris (1965) la menciona como dudosa para Argentina. En esta contribución se las describe e ilustra.

Se presenta una clave para la identificación de las nueve especies nativas del género, se comparan sus áreas de distribución en el país y su fenología.

\section{Arrabidaea samydoïdes (Cham.) Sandwith} Fig. 1

Sandwith N.Y., Kew Bull. 22(3): 413. 1968.

Bignonia samydoïdes Cham., Linnaea 7: 669. 1832.

Petastoma samydoides (Cham.) Miers, Proc.Roy. Hort. Soc. London 3: 195. 1863. Bureau E. y K. Schumann en Martius C., Fl. bras. 8(2): 78-79, tab. 78. 1896.

Liana con ramas cilíndricas, tomentosas, las más viejas lenticeladas. Hojas inferiores de cada rama simples, las apicales bifolioladas, zarcillo simple, caduco. Pecíolos y peciólulos tomentosos, ambos de 4-8 mm long. Folíolos ovados, obovados o latielípticos, 2-7 x 1,5-5,5 $\mathrm{cm}$, base cuneada o redondeada, ápice brevemente acuminado y mucronulado, agudo o redondeado, borde eroso, crenado-dentado o entero, pilosos en ambas caras, más en el envés, raramente glabros; domacios muy pequeños en el nacimiento de las venas secundarias, ocultos en el indumento. Flores en tirsos abiertos, ca. $8 \mathrm{~cm}$ long. Pedicelos 3-10 mm long. Cáliz 


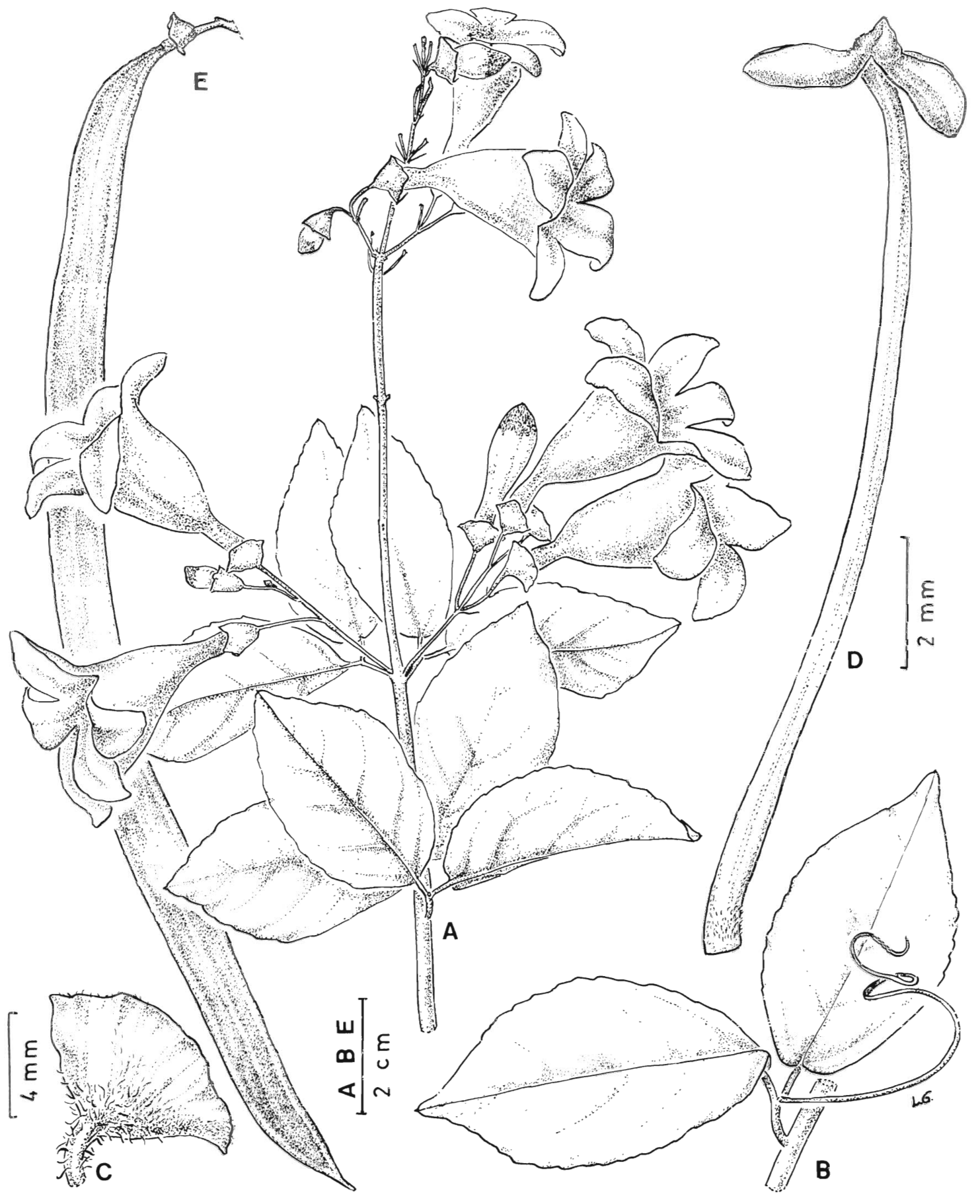

Fig. 1. Arrabidaea samydoïdes: A, Rama florífera. B, Hoja con zarcillo. C, Cáliz. D, Estambre. E, Fruto (Vanni et al 3538 , CTES).

anchamente campanulado, pateliforme, 3-5 x long., tubo glabro por fuera, lóbulos pilosos de 5-7,5 mm, con peios simples en la base, borde color notoriamente más claro, botones discodenticulado, piloso. Corola purpúrea, $3-5 \mathrm{~cm}$ loros, con el ápice marcadamente más claro en 
seco, debido a la pubescencia. Estambres didínamos, insertos en la corola, filamentos largos 10-20 mm iong., filamentos cortos 8-15 mm long., anteras divaricadas, tecas opuestas, conectivo apiculado, estaminodio $3 \mathrm{~mm}$ long. Ovario y disco 2,5-3 mm long., estilo 2,7-3 mm long., estigma elíptico, laminar, $2 \mathrm{~mm}$ long. Cápsula 18-25 x 1-1,4 cm, castaño-amarillenta, rebordes poco marcados.

Distribución y hábitat. Brasil (GO, MG, MS, PR, RJ, SP), Paraguay y Norte argentino (Salta y Misiones), en la selva.

Material estudiado: BRASIL. Mato Grosso do Sul: Rio Amambay, camino Ponta Porá - Amambay, 23 II 1968, Krapovickas et al. 14141 (BAA, CTES, G, LIL, LP). Minas Gerais: Serro, arredores, 17 III 1987, Hatschbach et al. 51038 (CTES, MBM); Poços de Caldas, Véu das Noivas, 19 I 1980, Krapovickas y Cristóbal 35394 (C, CTES, MBM, MO). Paraná: mun. Bocaiúva do Sul, Serra de Santana, 800m, 9 III 1993, Cordeiro \& Poliquesi 1023 (CTES, MBM); Sierra de São Luis, BR-277, 19 I 1985, Ferrucci et al. 293 (C, CTES, G, MO); mun. Rio Branco do Sul, Itarerama, 22 XI 1978, Hatschbach 41883 (CTES, MBM). ARGENTINA. Misiones: Dep. Iguazú, Parque Nacional Iguazú, paseos superiores, I 1996, Herrera y Toledo 134 (CTES); paseos superiores, salto Mbiguá, isla, 18 IX 1996 Herrera 201 (CTES); islas del Paseo Superior, 29 II 1996, Vanni et al. 3538 (CTES, MO). Salta: Dep. Orán, $15 \mathrm{Km}$ al oeste de Aguas Blancas, 600 m, 20 IV 1968, Fabris y Crisci 7316 (LP).

\section{Arrabidaea triplinervia (DC.) Baill. ex Bureau}

Fig. 2

Bureau, Vidensk.Meddel. Dansk Naturhist. Foren. Kjøbenhavn 99. 1893. Bureau y Schumann en Martius, Fl. bras. 8(2): 67-68. 1896.

Bignonia triplinervia DC. Prodr. 9: 153. 1845.

Liana con ramas estriadas longitudinalmente, densamente lenticeladas, nudos con pliegues interpeciolares, sin campos glandulares. Hojas bifolioladas, zarcillo simple. Pecíolo glabro, 10-22 mm long.; peciólulos pilosos. Folíolos elípticos, ovados o latiovados, generalmente acuminados, a veces obtusos, 3,5-11 x 2-8 cm, coriáceos, con escamas circulares es- parcidas, con tres venas importantes desde la base, haz lustrosa, envés con 1-2 pares de domacios grandes en el nacimiento de las venas laterales basales, a veces otros más pequeños en el nacimiento de las superiores, memorana del domacio con escamas por fuera, interiormente pilosa. Flores en tirso abierto (raci-mo de dicasios), 10-18 cm long. Cáliz tubuloso, 11-17 x 6-8 mm, borde denticulado. Corola infundibuliforme, 4,8-6,5 cm long., blanca, rosada hasta vinosa con lóbulos blanquecinos, pilosa por fuera. Estambres didínamos insertos en la corola; filamentos largos $18-21 \mathrm{~mm}$ long., filamentos cortos 13-16 mm long.; anteras divergentes, 3,5- 5,5 $\mathrm{mm}$ long., tecas rectas o arqueadas, conectivo de ápice retuso; estaminodio 2-7 $\mathrm{mm}$ long. Disco cupuliforme, $1 \mathrm{~mm}$ de alto. Ovario glabro, $4 \mathrm{~mm}$ long., estilo 28-35 $\mathrm{mm}$ long., estigma bífido. Cápsula $18 \times 1,2 \mathrm{~cm}$, valvas lignificadas, oscuras, rebordes poco marcados, vena media filiforme, no saliente.

Nombre vulgar: Isipó-y, isipó de agua (Latzina, 1937; Devoto \& Rothkugel, 1942).

Distribución y hábitat. Brasil (MG, MS, PR, RJ, SP), Bolivia y Paraguay, hasta el Parque Nacional Iguazú (Argentina, Misiones) donde vive en la selva.

Material estudiado. BRASIL. Mato Grosso do Sul: Ponto Alto, mun. Ponta Porã, 12 II 1983, Hatschbach 46149 (CTES, MBM). Paraná: S.Antonio da Platina, 30 III 1974, Kummrow 509 (CTES, MBM). BOLIVIA. Santa Cruz: Chiquitos, $3 \mathrm{Km}$ NE de Roboré, 59 $46^{\prime} \mathrm{W} 18^{\circ} 20^{\prime} \mathrm{S}, 300 \mathrm{~m}$ s.m., 22 IV 1980, Krapovickas y Schinini 36403 (CTES, MO). PARAGUAY. In regione fluminis Alto Paraná, 1909-10, Fiebrig 6144 (SI); Mbubebo IV, II 1922, Jörgensen 4178

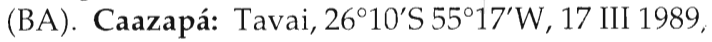
Soria 3360 (CTES, FCQ). Central: San Bernardino, III 1916, Rojas 1196 (CTES); Tarumandy, 2 IV 1973, Schinini 6203 (CTES, G, LiL, LP); 21 Km NW de San Bernardino, 9 V 1974, Schinini 9193 (CTES, G, LP). San Pedro: Ea. Alegría, III 1994, Soria 6540 (CTES, FCQ). ARGENTINA. Misiones: Dep. Iguazú, Parque Nacional Iguazú: paseos superiores, salto Eva, 18 IX 1996, Herrera et al. 199 (CTES); circuitos Cataratas, 27 III 1996, Tressens et al. 5586 (CTES, LIL, MO); islas del paseo superior, 29 II 1996, Vanni et al. 3544 (CTES, GH, LIL, MO). 


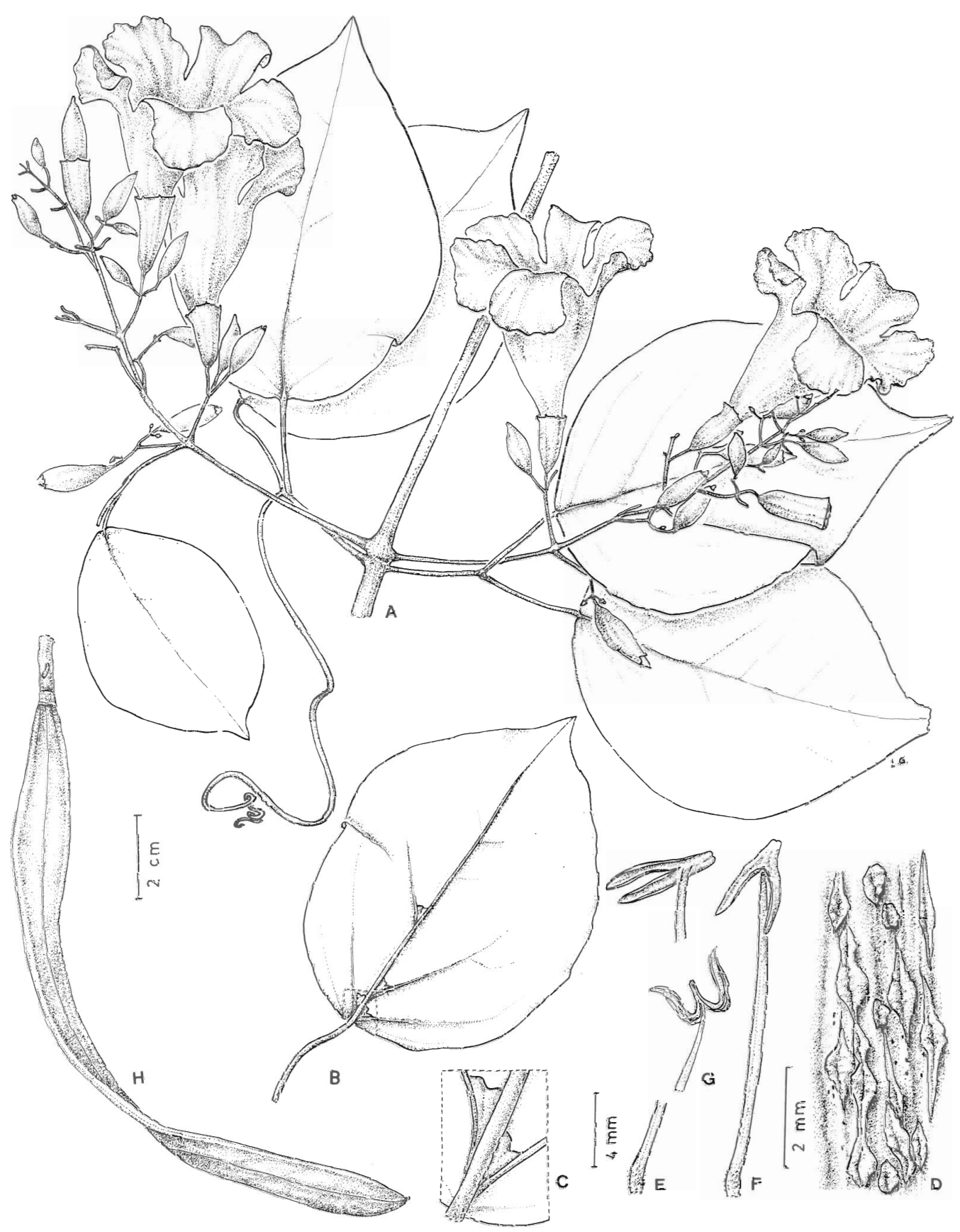

Fig. 2. Arrabidaea triplinervia: A, Rama florifera. B, Folíolo, envés. C, Detalle de domacios. D, Tallo, lenticelas. E-F, Estambres. G, Antera. H, Fruto (A-C, E-F, Vanni et al. 3544; D, Hatschbach 46149; G, Tressens et al. 5586; H, Schinini 9193; CTES). 


\section{Clave para las especies argentinas de Arrabidaea}

1. Folíolos con tres venas importantes desde la base, envés con 1-2 pares de domacios grandes, visibles a simple vista, en el nacimiento de las venas laterales basales, a veces otros más pequeños en el nacimiento de las venas laterales superiores. Cáliz tubuloso, 11-17 mm long. Anteras divergentes, de tecas rectas o arqueadas. Cápsula $18 \times 1,2 \mathrm{~cm}$, valvas lignificadas, oscuras, rebordes poco marcados, vena media filiforme no saliente

A. triplinervia (DC.) Baill. ex Bureau

1. Folíolos pinnatinervios, con domacios pequeños o sin elíos. Anteras divaricadas, de tecas opuestas (a veces divergentes en $A$. truncata).

2. Corola exteriormente glabra, excepto los lóbulos pubescentes; botones discoloros, ápice marcadamente más claro en seco. Folíolos con haz y envés pubescentes. Cápsula 18-25 x 1-1,4 cm, castaño-amarillenta, vena media no prominente ............................................................. A. samydoides (Cham.) Sandwith

2. Corola exteriormente pilosa excepto la porción basal del tubo, botones concoloros. Folíolos glabros o pubescentes.

3. Corola pequeña, menos de $2 \mathrm{~cm}$ long., blanca (excepcionalmente rosada). Folíolos con envés escamoso, sin axilas barbadas o domacios. Cápsula 11-22 x 0,7-1 cm, con rebordes, vena media filiforme ligeramente elevada ............................................................................ A. florida DC.

3. Corola de más de $2 \mathrm{~cm}$ long.

4. Corola blanca. Folíolos con o sin axilas barbadas o pequeños domacios en el envés. Cápsula cartácea, 13-17 x 0,8-1 cm, amarillenta en seco, sin rebordes, vena media filiforme, ligeramente saliente. Distrito chaqueño

A. truncata (Sprague) Sandwith

4. Corola rosada, rosado-lila o rosado purpúrea (excepcionalmente blanca).

5. Folíolos rojizos en herbario (a veces color café), elípticos u ovados, glabros, sin domacios; cáliz breve, tomentoso, con glándulas conspicuas cerca del borde superior. Cápsula 9-27 x $1 \mathrm{~cm}$, a veces con rebordes salientes, superficie granulosa, vena media filiforme ligeramente elevada ........................................................................... A. chica (Humb. et Bonpl.) Verl.

5. Folíolos no rojizos en herbario, cáliz con indumento variable.

6. Folíolos asimétricos, coriáceos, glabros, sin domacios. Cápsula 15-36 × 1,5-1,7 mm, vena media filiforme prominente, superficie rugosa

A. mutabilis Bureau et K.Schum.

6. Folíolos herbáceos o coriáceos, glabros o pubescentes especialmente en el envés.

7. Cáliz tubuloso, 7-10 mm long. Folíolos glabros, envés con axilas barbadas o domacios muy pequeños. Cápsula 12-25 x 1-1,3 cm, con rebordes, vena media filiforme ligeramente elevada ..................................................... caudigera (S.Moore) A.H.Gentry

7. Cáliz acampanado, tan largo como ancho. Folíolos glabros o pilosos.

8. Folíolos ovados o angustiovados, largamente acuminados, ápice no retuso, envés con axilas barbadas o domacios muy pequeños. Pseudoestípulas foliáceas. Cáliz de borde crespo irregularmente rasgado, raramente con glándulas. Cápsula 25-55 × 1,5$2 \mathrm{~cm}$, lenticelada, márgenes generalmente elevados y redondeados

A. selloi (Spreng.) Sandwith

8. Folíolos ovados, elípticos, a veces obovados, ápice retuso, envés con o sin axilas barbadas. Pseudoestípulas cortas, inconspicuas. Cáliz de borde y dientes pubérulos, con glándulas conspicuas. Cápsula 14-43 x 1,4-2 cm, plana, raramente con rebordes elevados, superficie punteada ...................................... A. corallina (Jacq.) Sandwith

\section{Distribución geográfica y hábitat}

Arrabidaea se extiende desde México y Antillas hasta el norte de Argentina. La localidad más austral donde fue coleccionado es Colón, provincia de Entre Ríos, situada a $32^{\circ} 10^{\circ}$ de lat. S.
Arrabidaea triplinervia y A. florida fueron encontradas solamente en Iguazú, en el extremo norte de Misiones. A. caudigera, A. chica y A. mutabilis viven en Misiones y Corrientes; de la última se conoce también un ejemplar coleccionado $25 \mathrm{~km}$ al $\mathrm{N}$ de Formosa, sobre la ruta 11. 
Tabla 1. Distribución de las especies de Arrabidaea en las provincias argentinas.

\begin{tabular}{|l|l|l|l|l|l|l|l|l|l|c|}
\hline & Jujuy & Salta & Tucum & S.Ester & Chaco & Formos & Sta.Fe & E.Rios & Ctes & Mnes. \\
\hline A. florida & & & & & & & & & & 8 \\
\hline A. triplinervia & & & & & & & & & & 3 \\
\hline A. caudigera & & & & & & & & & 12 & 46 \\
\hline A. chica & & & & & & & & & 6 & 95 \\
\hline A. mutabilis & & & & & & 1 & & & 6 & 23 \\
\hline A.samydoïdes & & 1 & & & & & & & & 3 \\
\hline A. selloi & 33 & 37 & & & & & & 5 & 1 & 66 \\
\hline A. truncata & 20 & 24 & 13 & 1 & 2 & 6 & & & & \\
\hline A. corallina & 54 & 55 & & & 72 & 40 & 1 & & 44 & \\
\hline
\end{tabular}

Los números indican la cantidad de ejemplares revisados.

Arrabidaea samydoüdes y $A$. selloi llegan a nuestro país por el noroeste y el nordeste. $A$. samiydoüdes fue recolectada en Salta (Aguas Blancas) y en Misiones (Iguazú). A. selloi vive en Jujuy y Salta, y va desde Misiones hasta Entre Ríos a través de Rio Grande do Sul y Uruguay; se conoce un ejemplar de Corrientes, sin localidad indicada, coleccionado por Muniez en 1928.

Arrabidaea truncata y A. corallina, tienen distribución amplia. A. truncata se extiende desde Jujuy hasta Formosa (Puerto Ramos, dep. Pilcomayo) hacia el este, y hasta Tucumán (Cruz Alta) hacia el sur. A. corallina se encuentra desde Jujuy hasta Corrientes y extremo norte de Santa Fe. Según Fabris (1965) A. corallina llega hasta Misiones y Entre Ríos, pero se comprobó que los ejemplares citados de estas provincias corresponden a $A$. selloi.

La distribución de las especies de Arrabidaea coincide en gran parte con las regiones fitogeográficas argentinas (Cabrera, 1976), hay dos grupos de especies: uno asociado al Dominio Amazónico, y otro al Chaqueño. Las siete especies mencionadas primero corresponden al Dominio Amazónico, cinco viven en las selvas mixtas de la Provincia Paranaense. A. selloi y A. samydoüdes se encuentran tanto en dicha provincia como en la selva de transición de la Provincia de Yungas.

Las últimas dos especies, A, truncata y $A$. corallina, están obviamente asociadas con la Provincia Chaqueña del Dominio Chaqueño, donde viven en bosques xerófilos y sabanas de los Distritos Chaqueños oriental y occidental.

Este análisis indica también, en cierta forma, la frecuencia de cada especie en nuestro país. A. corallina es una de las especies más extendidas, y la más frecuente. Le siguen $A$. selloi y $A$. chica. Las especies más raras son $A$. triplinervia y A. samydoüdes.

\section{Fenología}

Gentry (1974a) estableció cinco tipos fenológicos básicos en las Bignoniáceas centroamericanas. Según este autor las especies de Arrabidaea se encuadran en el Tipo 3: «cornuco- 
pia», que es la estrategia floral más generalizada en la familia. Este tipo de fenología se caracteriza por presentar un ciclo floral por año, de unas pocas semanas o prolongado, estacionalidad marcada, flores agrupadas en inflorescencias densas y numerosas, localizadas en el estrato superior del bosque o de la selva. Las especies con esta fenología atraen un espectro variado de polinizadores, entre los que predominan varios tipos de abejas.

Entre las Bignoniaceae centroamericanas hay varias especies de Arrabidaea, de las cuales tres llegan hasta Argentina: A. chica, A. corallina y A. florida. Gentry (1974b) hizo un estudio comparativo de la floración, recopilando los datos de los ejemplares de herbario recolectados en el área, y encontró que cada especie tiene estacionalidad propia, de manera que cada una presenta el pico de floración en un mes diferente.

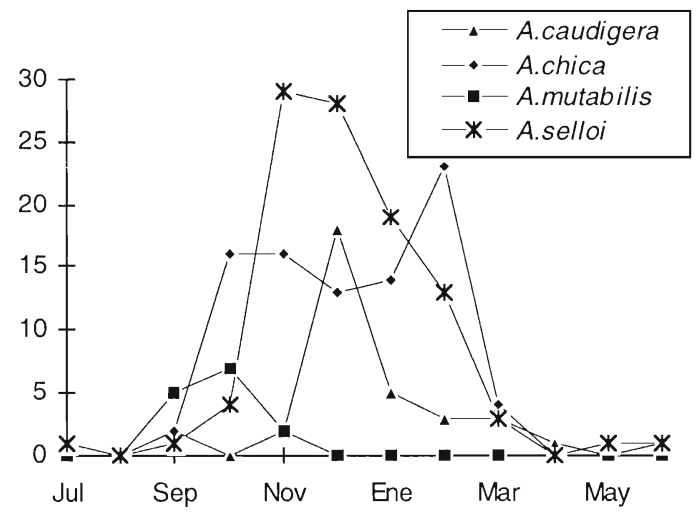

Gráfico 1. Floración de especies argentinas de Arrabidaea nativas del Dominio Amazónico.

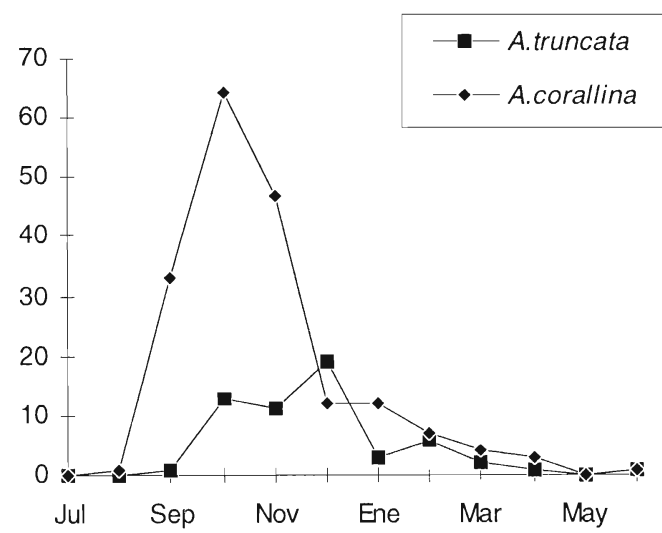

Gráfico 2. Floración de especies argentinas de Arrabidaea nativas del Dominio Chaqueño.
Se observa algo similar haciendo el mismo análisis sobre el material de las especies nativas de Arrabidaea conservado en los principales herbarios argentinos (BA, BAB, BAF, CORD, CTES, LIL, LP, SI). Se consideran seis de las nueve especies, pues de las tres restantes (A. florida, A. samydoides y A. triplinervia), solamente se coleccionaron unos pocos especímenes en Argentina.

En el gráfico 1 está representada la floración de las especies del Dominio Amazónico. En la ordenada se indica el número de ejemplares floríferos. A. mutabilis es primaveral, con el pico de floración en octubre. La mayor parte de los ejemplares en flor de A. caudigera (Gentry, 1976) son de diciembre. El período de floración de $A$. chica se extiende de septiembre a marzo y muestra dos picos, siendo más importante el de febrero. A. selloi vive en el noroeste y en el nordeste; en el noroeste la mayoría de los ejemplares floríferos son de noviembre (23), mientras en el nordeste son de diciembre (19), coincidiendo allí su pico de floración con el de $A$. caudigera.

En el gráfico 2 está representada la floración de las especies del Dominio Chaqueño. El pico de floración de $A$. corallina ocurre en octubre, mientras el de $A$. truncata tiene lugar en diciembre.

En Argentina la floración de Arrabidaea se extiende desde septiembre hasta abril, siendo excepcional en los meses restantes. Las especies simpátricas generalmente muestran sus picos de floración en meses diferentes.

\section{Agradecimientos}

A los árbitros cuyas observaciones contribuyeron a mejorar la presentación de este trabajo. Los viajes para estudiar el material de los herbarios argentinos fueron financiados por el programa PROFLORA (CONICET) y por la Secretaría Gral. de Ciencia y Técnica de la UNNE.

\section{Bibliografia}

CABRERA, A.L. 1976. Regiones fitogeográficas argentinas. Encicl.Arg.Agric.y Jard. 2(1): 1-85.

DEVOTO, F.E. \& M. ROTHKUGEL 1942. Indice de la flora leñosa argentina. Publ. Misc. Minist. Agric. 140. 183 
págs.

FABRIS, H.A. 1965. Flora Argentina: Bignoniaceae. Revista Mus.La Plata, Secc.Bot. 9(43): 273-419.

GENTRY, A.H. 1974a. Coevolutionary patterns in Central American Bignoniaceae. Ann.Missouri Bot.Gard. 61: 728-759.

- 1974b. Flowering Phenology and Diversity in Tropical Bignoniaceae. Biotropica 6: 64-68.
— 1976. Studies in Bignoniaceae 18: Notes on S.Moore's Mato Grosso Bignoniaceae, Ann.Missouri Bot.Gard. 63(1): 42-45.

LATZINA, E. 1937. Index de la Flora dendrológica argentina. Lilloa 1: 95-248.

SANDWITH, N.Y. 1968. Notes on Bignoniaceae: XXIX: Arrabidaea in Martius's «Flora Brasiliensis» and subsequently. Kew Bull. 22(3): 403-420. 